\title{
Advancing our understanding of ecological stability
}

Sonia Kéfi ${ }^{* 1}$, Virginia Dominguez-Garcia ${ }^{* 1}$, Ian Donohue ${ }^{2}$, Colin Fontaine ${ }^{3}$, Elisa Thébault ${ }^{4}$, Vasilis Dakos ${ }^{1}$

*co first-authors

${ }^{1}$ ISEM, CNRS, Univ. Montpellier, EPHE, IRD, Montpellier, France

${ }^{2}$ Department of Zoology, School of Natural Sciences, Trinity College Dublin, Dublin 2, Ireland

${ }^{3}$ UMR 7204, MNHN-CNRS-UPMC, CESCO, Paris, France

${ }^{4}$ CNRS, Sorbonne Université, Institute of Ecology and Environmental Sciences of Paris, 75005 Paris, France

\section{Email addresses:}

sonia.kefi@umontpellier.fr, virginia.dominguez-garcia@umontpellier.fr, donohui@tcd.ie, elisa.thebault@upmc.fr, colin.fontaine@mnhn.fr, vasilis.dakos@,umontpellier.fr

Statement of authorship: SK, VDo and VDa developed the original idea, SK, VDo, CF, ET and VDa performed the literature analysis, SK, VDo and CF analyzed the results, SK wrote the first full draft of the paper with the help of all co-authors.

Data accessibility statement: Should the manuscript be accepted, the data supporting the results will be archived in an appropriate public repository and the data DOI will be included at the end of the article.

Short running title: Ecological stability

Keywords: resilience, persistence, resistance, robustness, recovery, permanence, variability

Type of article: Ideas and Perspectives

Number of words in the abstract: 172

Number of words in the main text: 3938

Number of references: 66

3 figures in main text

Corresponding author:

Sonia Kéfi

Institut des Sciences de l'Evolution de Montpellier, Université de Montpellier, CC065, Bat 22, 33095

Montpellier cedex 05

Sonia.kefi@umontpellier.fr

$+33467144081$

Acknowledgements:

VD-G and SK were funded by the ANR project ARSENIC (ANR-14-CE02-0012)

ET and CF were funded by the ANR project ECOSTAB (ANR-17-CE32-0002/ECOSTAB). 


\begin{abstract}
The concept of ecological stability occupies a prominent place in both fundamental and applied ecological research. We review decades of work on the topic and examine how our understanding has progressed. We show that our understanding of stability has remained fragmented and is limited largely to simple or simplified systems. There has been a profusion of metrics proposed to quantify stability, of which only a handful are used commonly. Furthermore, studies typically quantify one to two metrics of stability at a time and in response to a single perturbation, with some of the main environmental pressures of today being the least studied. We argue that we need to build on the existing consensus and strong theoretical foundation of the stability concept to better understand its multidimensionality and the interdependencies between metrics, levels of organization and types of perturbations. Only by doing so can we make progress in the quantification of stability in theory and in practice and eventually build a more comprehensive understanding of how ecosystems will respond to ongoing environmental change.
\end{abstract}




\section{Introduction}

What underpins the apparent stability of natural systems? This question has been a core focus of ecologists since the very emergence of the discipline, spurring the parallel development of theory and empirical exploration to a far greater extent than many other topics in the field (Donohue et al. 2016). While early work explored fluctuations in population dynamics (Lotka 1925; Volterra 1926), later work evolved to a strong focus on the role of diversity for stability (Odum 1953; MacArthur 1955; Elton 1958; May 1973). The relationship between diversity and stability has continued to engage ecologists ever since (Ives \& Carpenter 2007; Allesina \& Tang 2012; Hautier et al. 2014; Johnson et al. 2014; Pennekamp et al. 2018), but the growing awareness of the extent of human-induced global change has precipitated exploration of whether and how ecological systems will be able to withstand and recover from intensifying environmental stresses (Holling 1973; May 1977; Pimm 1991; Scheffer et al. 2001).

The relatively simple intuitive meaning of stability contrasts with the multiplicity of ways it has been evaluated in theoretical and empirical studies. Grimm and Wissel (1997), for example, identified 163 definitions, 70 different stability concepts and more than 40 measures of stability in the literature. Some of these measures have been more used than others with a clear distinction between disciplines and approaches (Donohue et al. 2016). Nonetheless, the profusion of metrics together with the misuse of language have fragmented our understanding of ecological stability and jeopardized our ability to make progress in its study.

It has long been acknowledged that there is no stability per se but rather that stability is a multifaceted concept characterized by different properties (Supp. Fig. S1; Lewontin 1969; Orians 1975; Pimm 1984; Grimm \& Wissel 1997; Ives \& Carpenter 2007; Donohue et al. 2013, 2016). Recurrent attempts at characterizing these properties have helped organise our 
conceptualisation of stability immensely (Supp. Fig. S1), but the difficulty in doing so has also opened new questions. First, the multifaceted nature of stability cautions against the use of only some metrics without knowing more about how these metrics are interrelated at the risk of misevaluating the stability of the system (Harrison 1979; Loreau 1994; Donohue et al. 2013; Arnoldi et al. 2016; Arnoldi \& Haegeman 2016). Second, stability's assessment might depend on the organizational scale at which stability is measured (e.g. species versus total community; Tilman 1996). Third, the concept of stability is intricately related to that of perturbations (Donohue et al. 2013), which vary in their nature, temporal extent and spatial scale. Understanding the stability of ecological systems requires addressing these different aspects of stability - the multiple metrics, the various scales of organization, and the diverse perturbations - to investigate their possible interdependencies and relative relevance. Indeed, the question of how to assess the stability of complex ecological systems - What needs to be measured? At what scale? And how does stability scale up across different organizational levels? - remains largely open.

Here, we review how stability has been assessed in the ecological literature - theoretical and empirical - since the $1950 \mathrm{~s}$. We recorded the stability metrics used, the variables on which they were quantified, and the perturbations considered. We show that the evaluation of stability in ecological studies has been largely limited to using a single stability metric, on 'simple' species-poor systems, at a single organizational scale, in response to single perturbations. These observations raise a number of questions and challenges that we summarize in order to highlight where research is needed. 


\section{An overview of our understanding of stability}

To assess what has been done so far, we reviewed the ecological literature and recorded how stability was quantified in both theoretical and empirical papers. More specifically, we searched for the terms ecolog* AND stability, ecolog* AND resilience, ecolog* AND structural stability on the Web of Science in the period from 1900 to January 2018 in the journals Ecology, American Naturalist, Oikos, Ecology Letters, Science, Proceedings of the National Academy of Sciences of the United States of America (PNAS), Scientific Reports, Nature and Nature Communications.

This search retrieved 995 articles, from which we removed studies that did not explicitly measure, quantify, or calculate stability in any form (e.g. review or opinion papers). Our final database consisted of 459 entries (around $46 \%$ of the initial search). For each paper, we recorded: a) the type of study (theoretical, experimental/empirical (hereafter referred to as 'empirical') or both (hereafter referred to as 'mixed'), b) the system size (number of species or model dimension), c) the nature (what is perturbed), scale (who is affected: species, group of species, total community), and type (pulse, press, stochastic) of the perturbation(s) performed, and d) the nature (on what variable(s) are the stability metrics measured), scale (on which scale is the metric measured: species, group of species, total community), and type of metric measured. We merged metrics that were named differently in different studies despite having the same definition. The complete list of metrics recorded is summarized in Table S1.

\section{A proliferation of metrics}

The number of metrics used in theoretical and empirical studies has increased over time as new metrics keep being suggested in the literature - in papers published between 1970 and 1980, for example, there were 13 different metrics used, while about 34 different ones were used in 
papers published since 2010 (Fig. 1A). The vast majority of these metrics has received little use in the literature, while only a handful have been used broadly across studies (Fig. 1B). For example, coefficient of variation (CV) and resistance have both been used in more than 100 papers. The diversity of metrics used hinders comparisons among studies, since different metrics are not straightforward to compare. Moreover, the metrics used differ in theoretical versus empirical studies - dominant eigenvalue, the type of attractor (e.g. alternative stable states, dynamics becoming cyclic), $\mathrm{CV}$ and persistence are the most common metrics used in theoretical studies, whereas resistance and CV dominate in empirical studies (Table S1). This creates significant difficulties in linking theory with experiments and observations (Donohue et al. 2016).

These observations raise the question of what metrics to measure, and whether each metric provides complementary information or whether some of them are redundant. Papers typically quantify one to two metrics at a time (1.4 metrics on average in our database). This means that different metrics have rarely been quantified simultaneously and that we do not know much about how stability metrics relate to each other.

\section{A focus on 'simple' (species-poor) ecological systems}

The majority of experimental and theoretical studies identified in our literature search focused on relatively simple, species-poor systems. Among the empirical papers studied, $21 \%$ involved systems with less than 5 species, and 29\% with less than 10 (Supp. Fig. S2A). Among theoretical studies, 39\% studied systems with less than 5 species, while $45 \%$ focused on systems with less than 10 (Supp. Fig. S2B). 
Clearly, the studied systems are far less diverse and complex than most natural systems. This raises the question of whether our knowledge about the stability of simplified systems can be extrapolated to systems with more realistic levels of complexity.

\section{A bias towards measuring stability at the community scale (i.e. on aggregated variables)}

Stability can be measured at each of the various levels of ecological organization, at the species level, at the functional group at the community scale, with aggregated measures such as total biomass or productivity (Fig. 2iii). In our literature analysis, we found a significant bias towards quantifying stability from properties aggregated at the level of the community or metacommunity (e.g. total biomass or total cover; $59 \%$ of studies, Fig. 2iii). Relatively few studies quantify stability at finer scales of organization (i.e. at the level of species or groups of species). Further, the comparison of stability measures performed at different organizational levels in the same system remains poorly studied: $82 \%$ of the studies of our database measure stability at only one scale, $16 \%$ perform measures at two scales and only $2 \%$ consider three different scales when assessing stability.

Because current research focuses on the stability of a single organization level at a time, these observations raise the question of how stability at a given level of organization scales to other levels (i.e. how stability depends on the level of organization).

\section{A restricted examination of perturbations}

The idea of stability is intimately linked with how a system responds to perturbations. Traditionally, two broad types of perturbations are distinguished in ecology: press and pulse (Fig. 2i) (Bender et al. 1984). Press perturbations are defined as continuous disturbances causing the abundance or density of species to change permanently, or as long as the 
perturbation is present (e.g. species extinctions or sustained fishing pressure). Pulse perturbations are defined as instantaneous and short-term disturbances causing a sudden change in species abundances or densities, such as extreme climatic events or fire events. Next to these two types of perturbations, the stability of a system can also be evaluated as its dynamical response to noise (typically sustained random fluctuations), which can take the form of either demographic (intrinsic to the biological system) or environmental (extrinsic to the system) stochasticity (May 1973; Turelli 1978).

Interestingly, we found that a significant portion of studies quantify the stability of ecological systems in the absence of an explicit perturbation (i.e. the 'null' perturbation type in Fig. 3 left). Rather, such studies tend to look at dynamical properties of the system under different conditions, such as contrasting levels of species richness or environmental variables (e.g. nutrients, grazing pressure). This includes most of the studies investigating the links between diversity and community or ecosystem stability (e.g. Yachi \& Loreau 1999; McCann 2000; Tilman et al. 2006; Downing et al. 2014), in which ecosystem variability over time is typically compared across different levels of species richness. These studies have contributed greatly to the understanding of the links between biodiversity and stability, but it is nonetheless unclear to what extent these results can help us understand the consequences of biodiversity loss in natural conditions. Indeed, the stability assessed from comparing communities of different diversity levels might not be equivalent to the stability of a community following species removal (Díaz et al. 2003). For example, potential cascading effects following extinctions are ignored when comparing diversity treatment levels, and in particular the sequential effects of species loss (i.e. the order in which species are lost). Zavaleta \& Hulvey (2004) showed that a realistic species loss scenario decreased more strongly grassland resistance to invasions compared to random species loss. 
In addition, perturbations of a different nature (e.g. temperature, nutrient availability, $\mathrm{CO}_{2}$, light, salinity, rainfall) have attracted different levels of attention (Fig. 3 right). We, for example, know little about 'spatial stressors', such as habitat destruction and fragmentation, even though they have been identified as critical elements of global change (Millennium Ecosystem Assessment 2005).

Although multiple types of disturbances acting simultaneously is a defining feature of global environmental change (e.g. Millenium Ecosystem Assessment 2005; Piggott et al. 2015), this has been largely neglected since studies typically study a system's response to one or two perturbations (1.4 perturbations studied per paper on average in our database; Donohue et al. 2016). This finding is consistent with previous work, which highlighted that most studies about the effects of global change on species and their interactions have focused on only one global change driver at a time, while interactive effects are often neglected (Tylianakis et al. 2008).

Moreover, different perturbations may affect all or only a subset of the components of a system (Fig. 2ii). For example, harvesting, fishing and hunting typically affect just one or a few species in a system, whereas climate change most likely affects all species in a given community, though probably not in the same way. In our literature review, studies considering perturbations or treatments affecting more than one scale of organization (i.e. species, group or community) at a time were very rare, suggesting that the interplay of perturbations affecting different scales of organization is remarkably understudied ( $92 \%$ of the studies applied perturbations or treatments at only one scale and less than $0.5 \%$ considered perturbations affecting more than two different scales of biological organization).

Ecologists have neglected some important aspects of disturbances affecting ecosystems. The consequent gaps in our knowledge have led to a remarkably poor understanding of the impacts of many of the most important elements of global change and their interactive effects. 


\section{Challenges and opportunities}

Our literature analysis reveals that stability has typically been assessed with one or a few metrics, in species-poor systems, at a given level of organization, after a single perturbation. These observations raise the worrying question of whether our understanding of stability extends beyond those restricted conditions. Addressing this remains a fundamental challenge that hinders progress toward a more holistic understanding of stability applicable to the natural world. In this section, we highlight some of the challenges that need to be overcome in order to build such understanding.

\section{(1) Elucidating the relationships between stability metrics}

Because many different metrics are used in the literature and because they have rarely been assessed simultaneously, a key question is whether they all convey different information or whether some are redundant with each other. Previous studies have proposed various categorizations of stability metrics into groups based on their definition; these groups have been referred to as 'facets', 'properties', 'meanings' or 'components' (e.g. Pimm 1984; Grimm et al. 1992; Grimm \& Wissel 1997; Ives \& Carpenter 2007; Supp. Fig. S1). Organizing stability metrics into groups is an important but clearly not an easy task. Although recent theoretical advances have started to unravel the mathematical relationships between some of the stability metrics as well as the conditions for these interdependencies (Loreau 1994; Arnoldi et al. 2016; Arnoldi \& Haegeman 2016; Haegeman et al. 2016; Radchuk et al. 2019), we are far from having a complete picture of the relationships between stability metrics. 
Donohue and colleagues (2013) have suggested a way forward by studying correlations between metrics to estimate what they refer to as 'the dimensionality' of stability. The underlying idea is that if different stability metrics are strongly correlated with each other, this suggests that they bear similar information and that only one (or a few) of them is needed to characterize the system's stability. Conversely, if all the metrics are independent from each other, they all carry different information and they may all be needed to assess the system's stability. In the same vein, another approach could consist in identifying groups of metrics that are redundant with each other within groups but largely independent from each other across groups. Such grouping of the metrics would significantly facilitate the quantification of stability (Donohue et al. 2013; Arnoldi et al. 2016). Research is, however, needed to identify which categorizations are more meaningful.

Better understanding of relationships among metrics could also help bridge the gaps between theoretical and empirical studies. Not only do the metrics used in these studies differ (Donohue et al. 2016; Table S1), but only $2 \%$ of the papers in our literature analysis combine theory and empirical measurements (Supplementary Fig. S2B). These important disparities between theoretical and empirical studies result in much of the theoretical advances remaining untested and hinder the mechanistic understanding of empirical studies and our capacity to generalize them. Although there have been some success stories of bridging the gaps between theory and empirical studies (Mazancourt et al. 2013; Sanders et al. 2015, 2018; Donohue et al. 2017), these remain rare.

\section{(2) Unraveling the interdependency of stability across scales of organization}

Because species influence each other through networks of interactions in natural communities, even localized perturbations can spread rapidly through the whole community (Mrowicki et al. 2016). This interdependency between organization levels raises the question of the level of 
organization (e.g. species, group of species, community; Fig. 2ii) at which stability metrics should be measured.

For example, how does stability at the species level relate to community stability and vice versa? Many theoretical and experimental studies have found destabilizing effects of diversity on variability at species level but stabilizing effects of diversity at community or ecosystem level (e.g. Tilman 1996; Thébault et al. 2005; Jiang \& Pu 2009; Gross et al. 2014). Such differences between the variability at species and community level result from the fact that variability at community level is not only determined by species variability but also by the synchrony of species temporal dynamics (Yachi \& Loreau 1999). If species have asynchronous responses to environmental changes, community variability may decline with increasing species richness even if species variability increases. While variability has been investigated at different levels of organization, this has very rarely been the case for other stability measures (but see e.g. Haegeman et al. (2016) who showed that asymptotic resilience at the aggregated community level was unrelated to that calculated at the corresponding species level).

Further, some stability metrics only reflect the response of a single (or a few) species, despite the fact that they are typically measured at the 'system' scale. For example, resilience (the asymptotic return rate to equilibrium after a pulse perturbation, i.e. the long-term return rate) is determined by rare species, while the short-term return rate is determined by abundant species (Arnoldi et al. 2018). In case of very uneven species abundance or biomass distributions, community variability might also be determined by the variability of the most abundant species (e.g. Thébault \& Loreau 2006). So, the knowledge of a few species could be enough to infer the stability of whole communities, and these few species could be used as indicator species for community wide responses, solong as they can be identified.

\section{(3) Accounting for the multidimensional nature of disturbances}


Despite the high diversity in the nature of the disturbances used in stability studies (Fig. 3), disturbances are nonetheless generally characterized as one-dimensional, affecting at most a couple of ecological scales and acting in isolation from each other. Few studies have focused on interactive effects of disturbances acting on more than one scale of biological organization (e.g. community vs species). The main reason for this is the difficulty in conducting experiments that include multiple disturbance effects. Donohue et al. $(2013,2016)$ describe how this largely one-dimensional perspective has led to confused communication about stability and to increased risks of significantly underestimating the impacts of perturbations. Multiple disturbances can indeed have synergistic or antagonistic effects. For example, in an increasingly hotter climate, the chance of wildfire events may also increase (Jolly et al. 2015). The combined detrimental effect of multiple disturbances are well exemplified by coral reefs that have been lost because of the synergistic effects of coastal eutrophication, global warming, and hurricane disturbances (Hughes et al. 2017). Clearly, the question of how to best integrate different types and properties of disturbances when studying the stability of a given system is a significant challenge. A general solution may lie in recently developed frameworks for incorporating both the strength and duration of combined pulse and press disturbances to better understand the stability of ecosystems such as shrublands (Ratajczak et al. 2017).

In the same way as multiple simultaneous disturbances are understudied in spite of their known relevance in the current context of global change, the study of certain specific perturbations have lagged far behind, in particular those due to habitat fragmentation or destruction (Fig. 3 right), even though these have clearly been identified as major ongoing pressures on species worldwide (Pimm \& Raven 2000; Foley et al. 2005; Millennium Ecosystem Assessment 2005). Recent studies have started tackling this issue. Using an original network approach and simulating the sequential removal of different habitats, Evans et al. (2013) identified key habitats whose degradation would lead to disproportional species losses. Blüthgen et al. (2016) 
showed that land use intensity in forests and grasslands could decrease the temporal variability of bird and bat communities in an empirical study, while Gravel et al. (2016) and Guelzow et al. (2017) highlighted the critical role played by dispersal and ecological connectivity in moderating stability. These studies suggest that a better integration of the spatial dimension of perturbations and ecosystems might be fruitful to understand stability, not only because many important ecological process related to stability have a spatial component, but also because considering perturbations at the landscape scale might be a way to study more realistic perturbation cocktails.

\section{Conclusion}

Reviewing the literature on ecological stability since the 1950's helped us identify three gaps of knowledge as key challenges. i) We need to better understand the relationships between stability metrics to clarify what needs to be measured and in what context. Among other things, this could help bridge gaps between empirical and theoretical studies, which have used different metrics for practical reasons. ii) We need to investigate how a given metric changes depending on the level of biological organization considered, but also how different metrics may best characterize certain levels of organization. iii) We need to focus on multiple types of perturbations and especially those that are common and known to affect ecological systems particularly strongly. Addressing these challenges means that we need to embrace the multidimensionality of the stability concept by studying several stability metrics and perturbations simultaneously, and this at different scales (temporal, spatial and organizational).

Nonetheless, we also found that in recent decades the number of papers tackling multiple metrics of stability simultaneously has increased, albeit slowly (Supp. Fig. S3A). Multiple 
perturbations and, to a lesser extent, perturbations affecting multiple scales have also attracted more recent attention (Supp. Fig. S3B, D). Finally, more papers are quantifying stability at several scales (Supp. Fig. S3C). These encouraging trends suggest that we are slowly starting to fill the gaps in our understanding of the stability of complex ecological systems.

Of course, these challenges are not the only gaps in our knowledge of the stability of ecological systems. For example, recent work has explored how stability changes from local to larger spatial scales (Peterson et al. 1998; Wang \& Loreau 2014, 2016) using invariability-area relationships (Wang et al. 2017). Moreover, a key challenge is to understand how ecoevolutionary dynamics, and in particular ability of ecological systems to adapt to changes through evolution, can affect ecological responses to stress and the stability of ecological systems (Orians 1975). A recent experiment has shown how evolution can influence species coexistence in a pondweed community (Hart et al. 2019), for example. Lastly, it remains a challenge to deal with non-equilibrium dynamics, oscillations or even alternative states, as most metrics of stability still assume an equilibrium viewpoint. Nonetheless, recent approaches are taking advantage of the increasing availability of data derived from remote-sensing or longterm monitoring to estimate ecological stability via proxys (Scheffer et al. 2009; Dakos et al. 2012; Kéfi et al. 2014) and to quantify nonlinear dynamics (Ushio et al. 2018). A challenge is to translate these approaches into practical and accessible tools for quantifying stability in real ecological systems.

When thinking about stability, the simple metaphor of a ball rolling in a landscape that stabilizes at the bottom of a valley comes to mind. This metaphor has served well its purpose for 'simple' (i.e. low dimensional) systems, where there is no ambiguity about the definition and choice of state variables, reference states and disturbances. Ecological systems are, however, clearly not simple (Grimm et al. 1992). In an ecological community composed of 
many species, the multiple levels of organization involved, the multiple types of perturbation and scales of the system's response imply multiple ways in which stability can be quantified and interpreted, depending on the variable measured and the type of perturbation applied. Moving towards such a multi-dimensional consideration of stability appears as a key ingredient currently missing to improve our understanding of the stability of natural systems. 


\section{References}

Allesina, S. \& Tang, S. (2012). Stability criteria for complex ecosystems. Nature, 483, 205208.

Arnoldi, J.-F., Bideault, A., Loreau, M. \& Haegeman, B. (2018). How ecosystems recover from pulse perturbations: A theory of short- to long-term responses - ScienceDirect. J. Theor. Biol., 436, 79-92.

Arnoldi, J.-F. \& Haegeman, B. (2016). Unifying dynamical and structural stability of equilibria. Proc. R. Soc. Lond. A, 472, 20150874.

Arnoldi, J.-F., Loreau, M. \& Haegeman, B. (2016). Resilience, reactivity and variability: A mathematical comparison of ecological stability measures. J. Theor. Biol., 389, 47-59.

Bender, E.A., Case, T.J. \& Gilpin, M.E. (1984). Perturbation Experiments in Community Ecology: Theory and Practice. Ecology, 65, 1-13.

Blüthgen, N., Simons, N.K., Jung, K., Prati, D., Renner, S.C., Boch, S., et al. (2016). Land use imperils plant and animal community stability through changes in asynchrony rather than diversity. Nat. Commun., 7, 10697.

Dakos, V., Carpenter, S.R., Brock, W.A., Ellison, A.M., Guttal, V., Ives, A.R., et al. (2012). Methods for Detecting Early Warnings of Critical Transitions in Time Series Illustrated Using Simulated Ecological Data. PLoS ONE, 7, e41010.

Díaz, S., Symstad, A.J., Stuart Chapin, F., Wardle, D.A. \& Huenneke, L.F. (2003). Functional diversity revealed by removal experiments. Trends Ecol. Evol., 18, 140-146.

Donohue, I., Hillebrand, H., Montoya, J.M., Petchey, O.L., Pimm, S.L., Fowler, M.S., et al. (2016). Navigating the complexity of ecological stability. Ecol. Lett., 19, 1172-1185.

Donohue, I., Petchey, O.L., Kéfi, S., Génin, A., Jackson, A.L., Yang, Q., et al. (2017). Loss of predator species, not intermediate consumers, triggers rapid and dramatic extinction cascades. Glob. Change Biol., 23, 2962-2972.

Donohue, I., Petchey, O.L., Montoya, J.M., Jackson, A.L., McNally, L., Viana, M., et al. (2013). On the dimensionality of ecological stability. Ecol. Lett., 16, 421-429.

Downing, A.L., Brown, B.L. \& Leibold, M.A. (2014). Multiple diversity-stability mechanisms enhance population and community stability in aquatic food webs. Ecology, 95, $173-184$.

Elton, C.S. (1958). Ecology of Invasions by Animals and Plants. Chapman \& Hall, London.

Evans, D.M., Pocock, M.J.O. \& Memmott, J. (2013). The robustness of a network of ecological networks to habitat loss. Ecol. Lett., 16, 844-852.

Gravel, D., Massol, F. \& Leibold, M.A. (2016). Stability and complexity in model metaecosystems. Nat. Commun., 7, 12457. 
Grimm, V., Schmidt, E. \& Wissel, C. (1992). On the application of stability concepts in ecology. Ecol. Model., 63, 142-161.

Grimm, V. \& Wissel, C. (1997). Babel, or the ecological stability discussions: an inventory and analysis of terminology and a guide for avoiding confusion. Oecologia, 323-334.

Gross, K., Cardinale, B.J., Fox, J.W., Gonzalez, A., Loreau, M., Wayne Polley, H., et al. (2014). Species Richness and the Temporal Stability of Biomass Production: A New Analysis of Recent Biodiversity Experiments. Am. Nat., 183, 1-12.

Guelzow, N., Muijsers, F., Ptacnik, R. \& Hillebrand, H. (2017). Functional and structural stability are linked in phytoplankton metacommunities of different connectivity. Ecography, 40, 719-732.

Haegeman, B., Arnoldi, J.-F., Wang, S., Mazancourt, C. de, Montoya, J.M. \& Loreau, M. (2016). Resilience, invariability, and ecological stability across levels of organization. bioRxiv, 085852 .

Harrison, G.W. (1979). Stability under Environmental Stress: Resistance, Resilience, Persistence, and Variability. Am. Nat., 113, 659-669.

Hart, S.P., Turcotte, M.M. \& Levine, J.M. (2019). Effects of rapid evolution on species coexistence. Proc. Natl. Acad. Sci., 116, 2112-2117.

Hautier, Y., Seabloom, E.W., Borer, E.T., Adler, P.B., Harpole, W.S., Hillebrand, H., et al. (2014). Eutrophication weakens stabilizing effects of diversity in natural grasslands. Nature, 508, 521-525.

Holling, C.S. (1973). Resilience and Stability of Ecological Systems. Annu. Rev. Ecol. Syst., $4,1-23$.

Hughes, T.P., Barnes, M.L., Bellwood, D.R., Cinner, J.E., Cumming, G.S., Jackson, J.B.C., et al. (2017). Coral reefs in the Anthropocene. Nature, 546, 82-90.

Ives, A.R. \& Carpenter, S.R. (2007). Stability and Diversity of Ecosystems. Science, 317, 5862.

Jiang, L. \& Pu, Z. (2009). Different Effects of Species Diversity on Temporal Stability in Single-Trophic and Multitrophic Communities. Am. Nat., 174, 651-659.

Johnson, S., Domínguez-García, V., Donetti, L. \& Muñoz, M.A. (2014). Trophic coherence determines food-web stability. Proc. Natl. Acad. Sci., 111, 17923-17928.

Jolly, W.M., Cochrane, M.A., Freeborn, P.H., Holden, Z.A., Brown, T.J., Williamson, G.J., et al. (2015). Climate-induced variations in global wildfire danger from 1979 to 2013. Nat. Commun., 6, 7537.

Kéfi, S., Guttal, V., Brock, W.A., Carpenter, S.R., Ellison, A.M., Livina, V.N., et al. (2014). Early Warning Signals of Ecological Transitions: Methods for Spatial Patterns. PLoS ONE, 9, e92097. 
Lewontin, R.C. (1969). The meaning of stability. Brookhaven Symp. Biol., 22, 13-24.

Loreau, M. (1994). Material Cycling and the Stability of Ecosystems. Am. Nat., 143, 508-513.

Lotka, A.J. (1925). Elements of Physical Biology. Williams and Wilkins Company.

MacArthur, R. (1955). Fluctuations of Animal Populations and a Measure of Community Stability. Ecology, 36, 533-536.

May, R.M. (1973). Stability and Complexity in Model Ecosystems. Princeton University Press.

May, R.M. (1977). Thresholds and breakpoints in ecosystems with a multiplicity of stable states. Nature, 269, 471-477.

Mazancourt, C. de, Isbell, F., Larocque, A., Berendse, F., Luca, E.D., Grace, J.B., et al. (2013). Predicting ecosystem stability from community composition and biodiversity. Ecol. Lett., 16, $617-625$.

McCann, K.S. (2000). The diversity-stability debate. Nature, 405, 228-233.

Millenium Ecosystem Assessment. (2005). Ecosystems and human well-being: desertification synthesis.

Millennium Ecosystem Assessment. (2005). Ecosystem and Human Well-being: Synthesis. Island Press, Wahington.

Mrowicki, R.J., O’Connor, N.E. \& Donohue, I. (2016). Temporal variability of a single population can determine the vulnerability of communities to perturbations. J. Ecol., 104, 887897.

Odum, E.P. (1953). Fundamentals of Ecology. Saunders, Philadelphia.

Orians, G.H. (1975). Diversity, stability and maturity in natural ecosystems. In: Unifying concepts in ecology. W.H. van Dobben \& R.H. Lowe-McDonnell, The Hague, The Netherlands, pp. 139-149.

Pennekamp, F., Pontarp, M., Tabi, A., Altermatt, F., Alther, R., Choffat, Y., et al. (2018). Biodiversity increases and decreases ecosystem stability. Nature, 563, 109.

Peterson, G., Allen, C.R. \& Holling, C.S. (1998). Ecological Resilience, Biodiversity, and Scale. Ecosystems, 1, 6-18.

Piggott, J.J., Salis, R.K., Lear, G., Townsend, C.R. \& Matthaei, C.D. (2015). Climate warming and agricultural stressors interact to determine stream periphyton community composition. Glob. Change Biol., 21, 206-222.

Pimm, S.L. (1984). The complexity and stability of ecosystems. Nature, 307, 321-326.

Pimm, S.L. (1991). The balance of nature?: Ecological issues in the conservation of species and communities. The University of Chicago Press. Chicago, IL, USA. 
Radchuk, V., Laender, F.D., Cabral, J.S., Boulangeat, I., Crawford, M., Bohn, F., et al. (2019). The dimensionality of stability depends on disturbance type. Ecol. Lett., 22, 674-684.

Ratajczak, Z., D’Odorico, P., Collins, S.L., Bestelmeyer, B.T., Isbell, F.I. \& Nippert, J.B. (2017). The interactive effects of press/pulse intensity and duration on regime shifts at multiple scales. Ecol. Monogr., 87, 198-218.

Sanders, D., Kehoe, R. \& van Veen, F.J.F. (2015). Experimental Evidence for the PopulationDynamic Mechanisms Underlying Extinction Cascades of Carnivores. Curr. Biol., 25, 31063109.

Sanders, D., Thébault, E., Kehoe, R. \& Veen, F.J.F. van. (2018). Trophic redundancy reduces vulnerability to extinction cascades. Proc. Natl. Acad. Sci., 115, 2419-2424.

Scheffer, M., Bascompte, J., Brock, W.A., Brovkin, V., Carpenter, S.R., Dakos, V., et al. (2009). Early-warning signals for critical transitions. Nature, 461, 53-59.

Scheffer, M., Carpenter, S., Foley, J.A., Folke, C. \& Walker, B. (2001). Catastrophic shifts in ecosystems. Nature, 413, 591-596.

Thébault, E. \& Loreau, M. (2006). The relationship between biodiversity and ecosystem functioning in food webs. Ecol. Res., 21, 17-25.

Thébault, E., Loreau, M., Morin, A.E.P.J. \& Losos, E.J.B. (2005). Trophic Interactions and the Relationship between Species Diversity and Ecosystem Stability. Am. Nat., 166, E95E114.

Tilman, D. (1996). Biodiversity: Population Versus Ecosystem Stability. Ecology, 77, 350363.

Tilman, D., Reich, P.B. \& Knops, J.M.H. (2006). Biodiversity and ecosystem stability in a decade-long grassland experiment. Nature, 441, 629-632.

Turelli, M. (1978). Does Environmental Variability Limit Niche Overlap? Proc. Natl. Acad. Sci. U.S. A., 75, 5085-5089.

Tylianakis, J.M., Didham, R.K., Bascompte, J. \& Wardle, D.A. (2008). Global change and species interactions in terrestrial ecosystems. Ecol. Lett., 11, 1351-1363.

Ushio, M., Hsieh, C., Masuda, R., Deyle, E.R., Ye, H., Chang, C.-W., et al. (2018). Fluctuating interaction network and time-varying stability of a natural fish community. Nature, 554, 360363.

Volterra, V. (1926). Fluctuations in the Abundance of a Species considered Mathematically. Nature, 118, 558-560.

Wang, S. \& Loreau, M. (2014). Ecosystem stability in space: $\alpha, \beta$ and $\gamma$ variability. Ecol. Lett., 17, 891-901.

Wang, S. \& Loreau, M. (2016). Biodiversity and ecosystem stability across scales in 
metacommunities. Ecol. Lett., 19, 510-518.

Wang, S., Loreau, M., Arnoldi, J.-F., Fang, J., Rahman, K.A., Tao, S., et al. (2017). An invariability-area relationship sheds new light on the spatial scaling of ecological stability. Nat. Commun., 8, 15211.

Yachi, S. \& Loreau, M. (1999). Biodiversity and Ecosystem Productivity in a Fluctuating Environment: The Insurance Hypothesis. Proc. Natl. Acad. Sci. U. S. A., 96, 1463-1468.

Zavaleta, E.S. \& Hulvey, K.B. (2004). Realistic Species Losses Disproportionately Reduce Grassland Resistance to Biological Invaders. Science, 306, 1175-1177. 


\section{Figures}

A

B
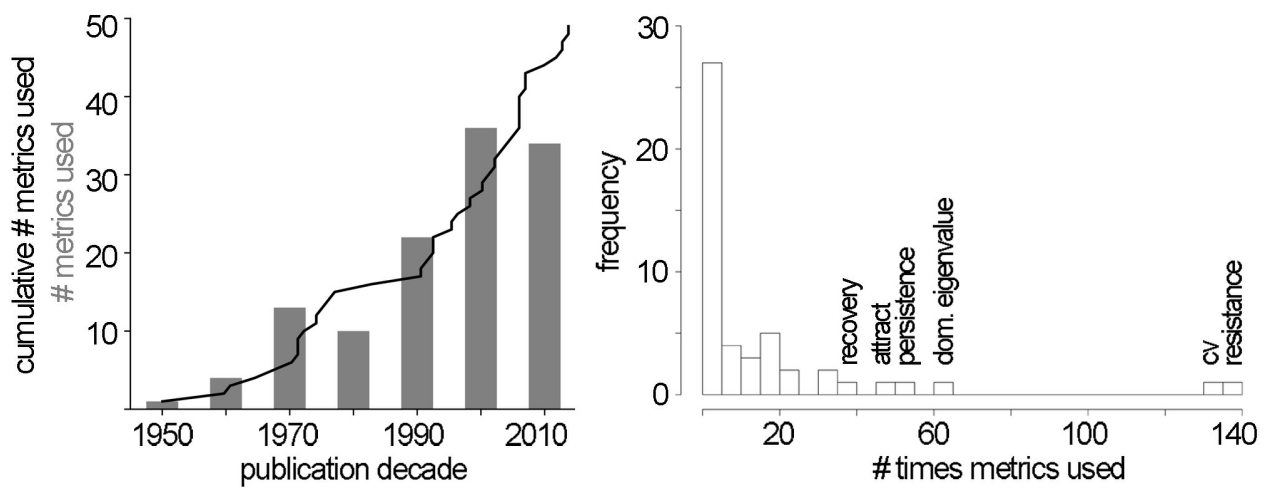

Figure 1. Metrics of stability. A) Grey bars represent the number of different metrics used in the papers of that decade (e.g. 14 metrics were used in the papers published between 1970 and 1979). The black line displays the cumulative number of metrics used in the papers until a given year (i.e. the total number of metrics used in the papers between 1950 and that year). B) Distribution of the number of times metrics have been used in the studies of our database (i.e. in how many papers; see Table S1 for a list of stability metrics).

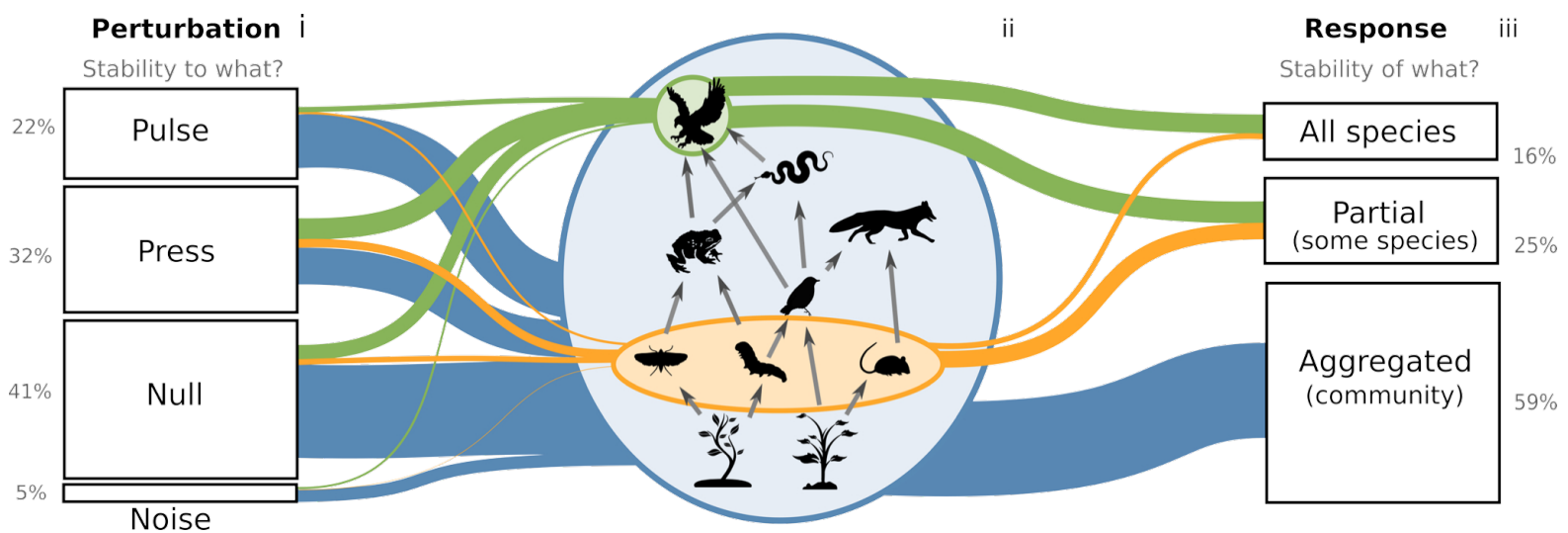

Figure 2. Assessing stability in ecological systems requires identifying i) the nature of the perturbation (the stability 'to what'?) [Pulse, Noise or Press], its intensity and direction, ii) the scale at which it affects the ecological system [individual species in green, groups of species (e.g. functional or trait-based) in orange, and whole system in blue] and iii) the scale at which the stability metrics is measured (the stability 'of what'?), which may differ from the scale at which the perturbation occurs. Stability metrics can be quantified on either each of all species of the system ('all species'), some species ('partial') or at the level of the whole community ('aggregated'). The percentages indicate the percentage of studies included in our database. Lines from the 
perturbation categories on the left indicate the proportion of studies with this type of perturbation applied on each level of organization.

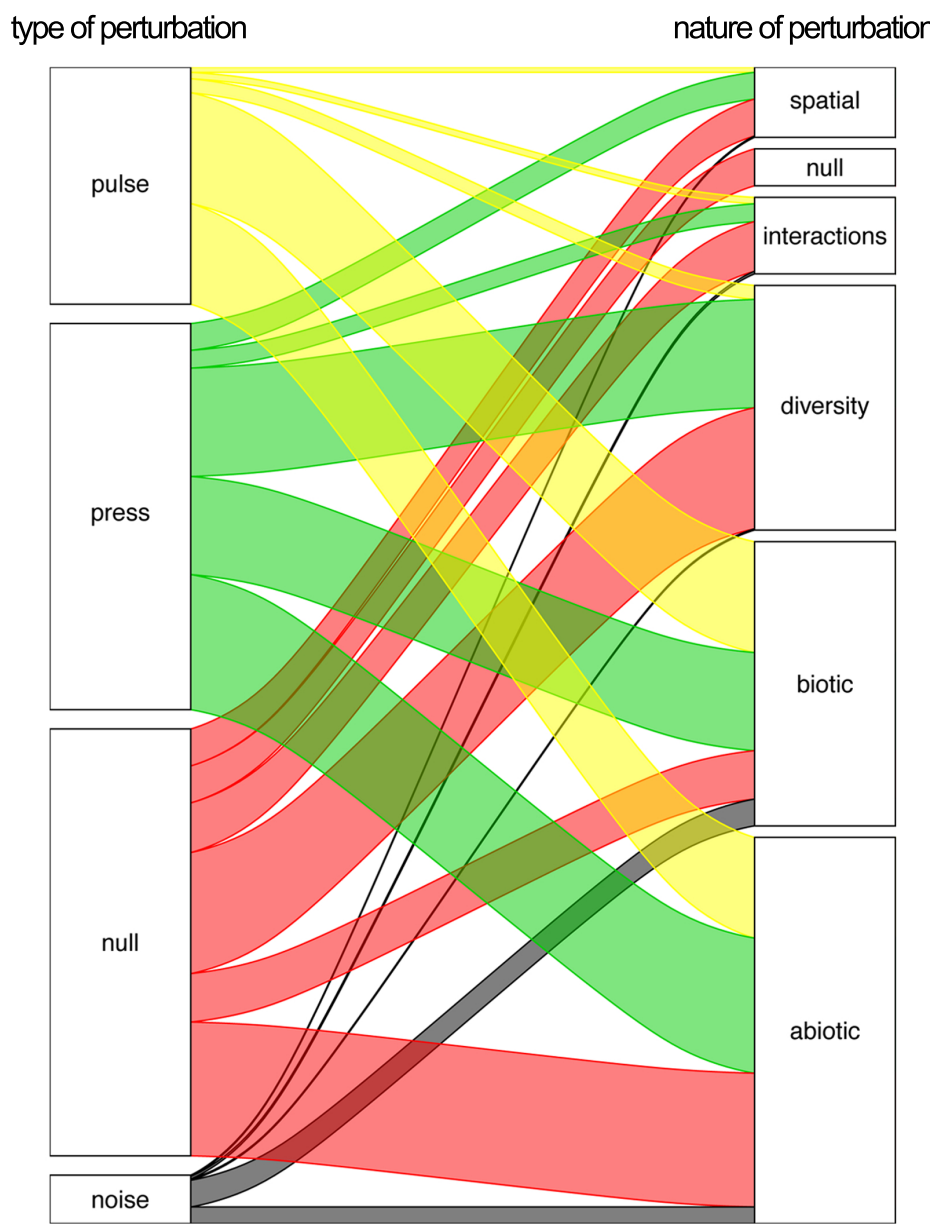

Figure 3. Alluvial plot of the type and nature of perturbations. The 'perturbation types' are defined in the 'A restricted examination of perturbations' part in the main text. 'Null' refers to studies that don't have an explicit perturbation but rather compare treatments (e.g. Biodiversity-Ecosystem Functioning studies or studies comparing persistence of different food web structures); in these cases, we still record the nature of the treatment (which is why there are flows between 'Null' and 'nature of perturbation'). Flow thickness between boxes is proportional to the number of papers, and colors reflect the type of perturbation. Regarding the 'nature of the perturbation': 'spatial' refers to cases where the amount, quality, structure or connectivity of the habitat were affected, 'Null' are the cases where nothing is changed, 'interactions' refers to situations where the interactions between species are changed, 'diversity' are cases where the number and/or identity of species are changed, 'biotic' includes cases where a biotic element other than species diversity is changed (e.g. biomass, abundance, productivity, cover, herbivory), and 'abiotic' refers to cases where an abiotic condition is changed (e.g. pH, temperature). 


\section{Supplementary material}

Table S1. List of metrics. Metrics found in the literature review to quantify or characterize stability, a brief description and the number of times they were found in total, in mixed, empirical and theoretical papers. For readability, the metrics were roughly organized based on the general question they address (last column on the right): i) How variable is the system in time or space? (constancy), ii) Whether and how fast a system recovers following a perturbation (recovery), iii) How much a system changes in the presence of disturbance compared to reference undisturbed conditions? (resistance), iv) How likely is the system to change state, and what states can be reached? (dynamical attractors). Note that the metrics were categorized based on their definition (such as stated in column 'metrics') and that the code name mentioned in the column 'metrics code' may therefore not correspond to the name of the metrics used in the papers studied. 


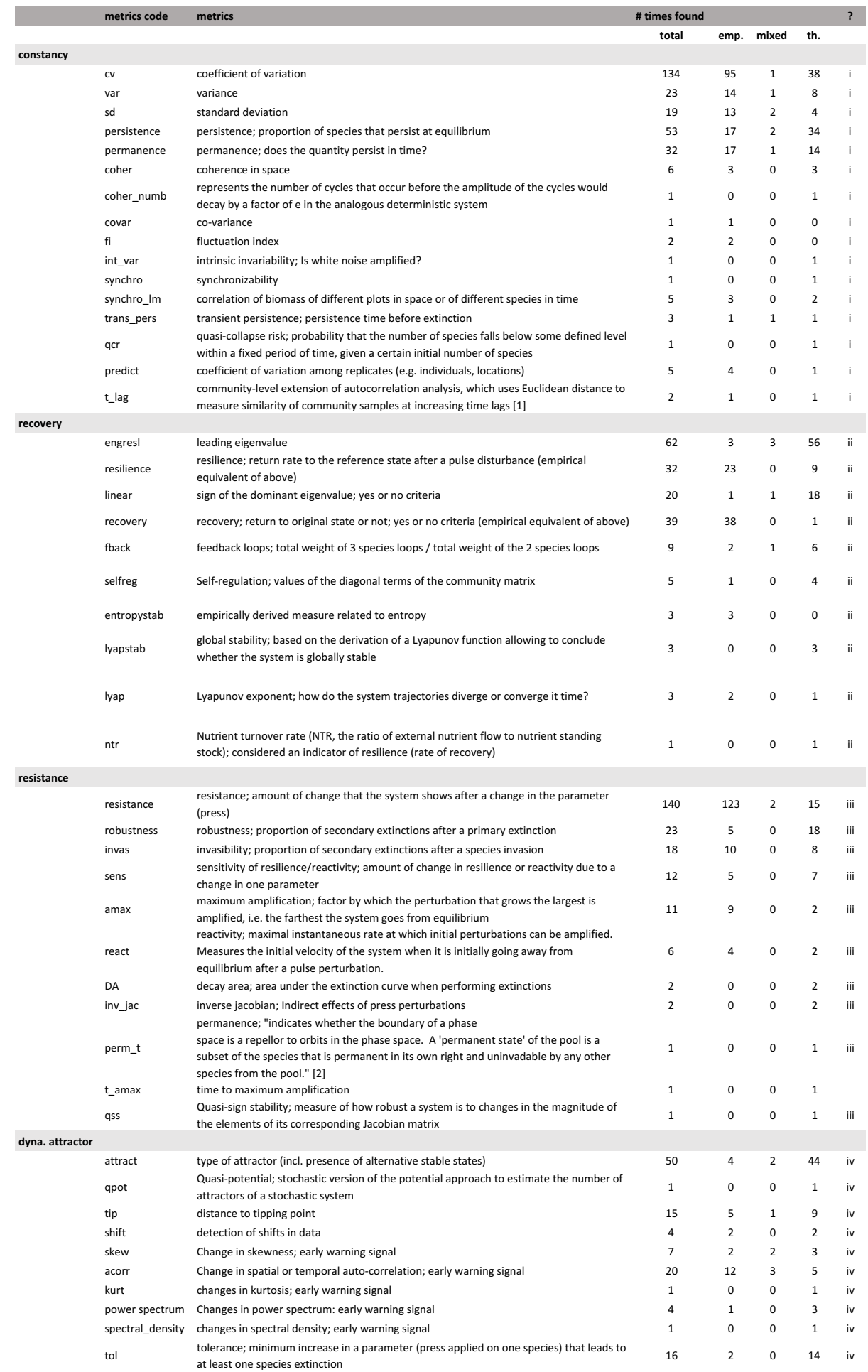




\section{Supplementary figures}

Stability properties

\begin{tabular}{|c|c|c|c|}
\hline $1970 s$ & $1980 s$ & 1990s & $2000 s$ \\
\hline Constancy & Stable & Constancy & \\
\hline Persistence & $\begin{array}{l}\text { Persistence } \\
\text { Variability }\end{array}$ & Persistence & $\begin{array}{l}\text { Persistence } \\
\text { Variability }\end{array}$ \\
\hline Inertia -- & Resistance & Resistance & \\
\hline Elasticity & Resilience & Resilience & \\
\hline Amplitude & & Domain of Attraction & Alternative States \\
\hline Cyclical Stability -- & & & \\
\hline Trajectory Stability & & & Nonpoint Attractors \\
\hline (Orian 1975) & (Pimm 1984) & (Grimm \& Wissel 1997) & (Ives \& Carpenter 2007) \\
\hline
\end{tabular}

Supp. Fig. S1. Groups of stability metrics, or stability 'properties', as proposed in four seminal review papers since the 50ies. Almost every decade a seminal review paper has attempted to organize stability metrics into categories, also referred to as 'facets', 'concepts', 'properties', 'aspects' or 'components'. These reviews reveal that ecologists have been strikingly consistent in their description of ecological stability over time. It is interesting to note that the terms used for these categories have partly changed, but consistently refer to similar characteristics (see table below). As discussed in these reviews, different categories of stability describe different ecosystem properties and have therefore led to multiple diversity-stability relationships, to different conclusions on the relative stability of communities, and to differences in quantifying the sensitivity of ecosystems to stress. 
A

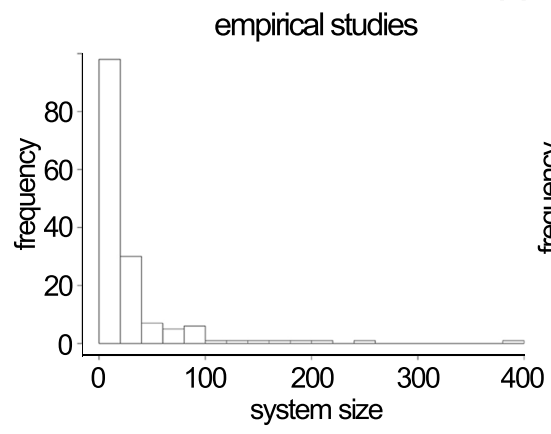

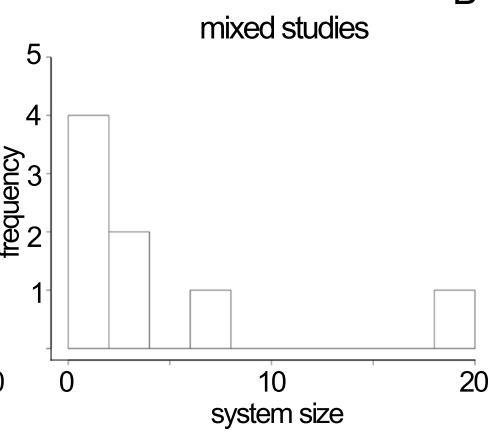

B

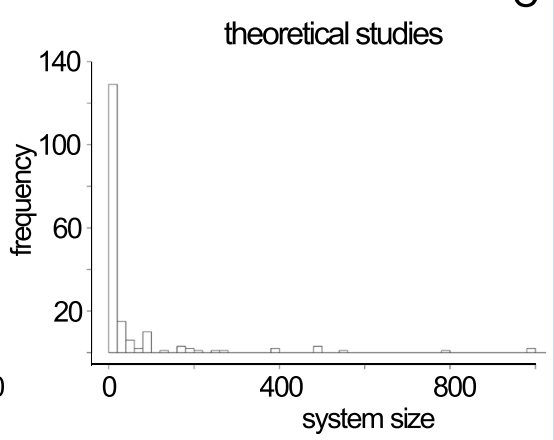

Supp. Fig. S2. Distribution of the size of the systems studied (i.e. number of species, groups of species or dynamical equations) in empirical, mixed and theoretical studies.

A

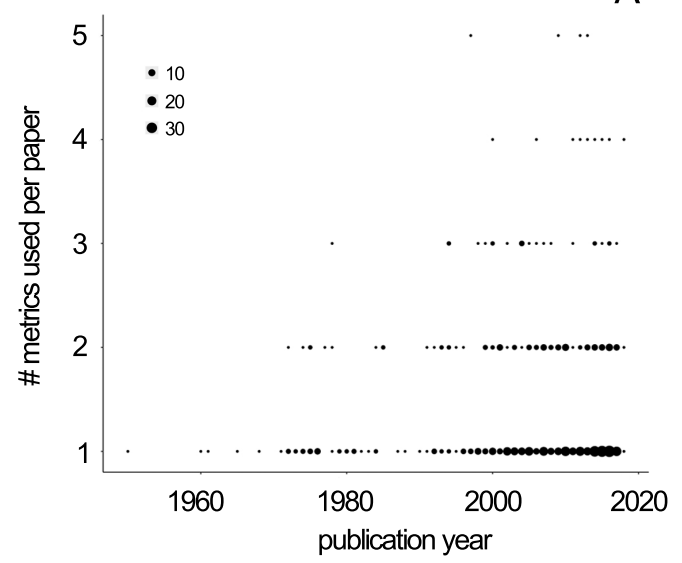

C

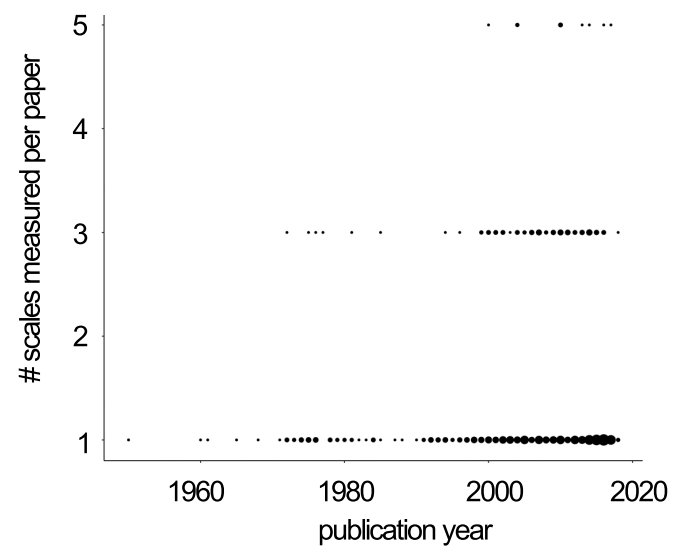

B

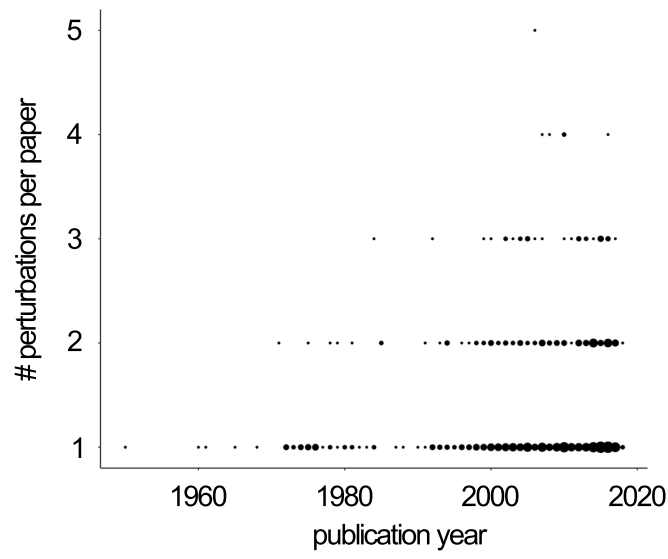

D

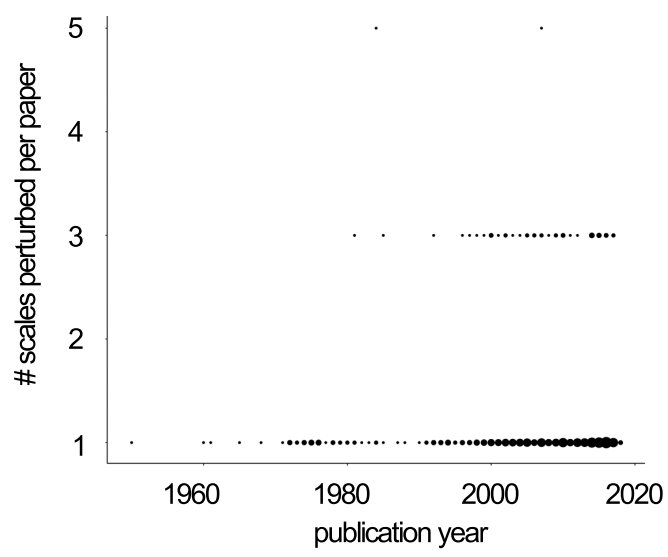

Supp. Fig. S3. Progress in our multidimensional description of stability across years. A) Number of metrics used in a given paper over time. B) Number of perturbations studied per paper as a function of the publication year. C) Number of scales (i.e. species, group of species or community) on which stability metrics are evaluated per paper as a function of publication year. D) Number of scales affected by a perturbation per paper as a function of 
publication year. A-D) Size of the dots are proportional to the number of papers involved (see insert in panel A for a legend). 